\title{
Minisuíno (minipig) na pesquisa biomédica experimental. O Minipig br1
}

Mario Mariano*

\begin{abstract}
Mariano M. Minisuíno (minipig) na pesquisa biomédica experimental: o Minipig brl. Acta Cir Bras [serial online] 2003 Set-Out;18(5). Disponível em URL: http://www.scielo.br/acb.

RESUMO - Diferentes espécies de animais vertebrados e invertebrados têm sido usados em investigação biomédica experimental. Neste trabalho é apresentada uma breve revisão sobre o uso do suíno em pesquisa biomedica e sobre as similaridades da anatomia, fisiologia, bioquímica e imunologia desta espécie com aquelas do homem. É também revisto o desenvolvimento do minipig nos anos 60 e os sucessos obtidos com este modelo animal nas mais diversas áreas da medicina experimental. Finalmente, são descritas as características básicas de uma colônia de minipigs desenvolvida pelo autor (Minipig brl). São descritas as condições de alimentação desses animais, como são mantidos, vacinados e selecionados. Pesam de 10-15 quilos com seis meses de idade e de 30-37 quilos com onze meses. A possível contribuição desse modelo animal para o desenvolvimento da cirurgia experimental e para outras áreas de experimentação animal no país é discutida.
\end{abstract}

DESCRITORES - Animais de laboratório. Suínos. Miniporco. Minipig.

\section{O suíno na Medicina}

Desde os primórdios da Medicina até os dias atuais, as mais diferentes espécies de animais vertebrados e invertebrados têm sido utilizadas como objeto de experimentação. Essa prática continua sendo largamente empregada com o intuito de se desenvolver métodos e técnicas que possam gerar instrumentos de "cura" ou alívio para os males que acometem o homem e outros animais.

Dentre as várias espécies utilizadas em experimentação biomédica destaca-se o porco (Sus scropha). Como citado por Swinddle (1983) ${ }^{1}$, o uso do suíno em pesquisas científicas é uma prática antiga. Nos anos de 1540, Versalius, em sua famosa obra $D e$ Humani Corporis Fabrica, em que tentava descrever os mecanismos que regem o corpo humano; desenhou um porco sendo utilizado em experimentação da maneira como o médico grego Galeno o fizera há mais de mil anos antes. Segundo o autor essa deve ser a mais antiga ilustração do uso desses animais nas diferentes áreas da ciência.

Em 1628, Willian Havey que descreveu os mecanismos que regem a circulação sangüínea, também utilizou o suíno como animal de experimentação para melhor compreender a fisiologia humana. Segundo Swindle $e$ col. (1992) ${ }^{2}$ esses estudos embasaram as normas da medicina experimental por Claude Bernard 250 anos após, normas essas que regem os procedimentos experimentais em biologia e medicina de nossos dias.

\section{O porco na experimentação animal e suas limitações}

Estão hoje amplamente estabelecidas na literatura as semelhanças entre a anatomia, fisiologia e fisiopatologia dos suínos e as do homem (Bustard and

* Prof. Titular do Departamento de Patologia, Faculdade de Medicina Veterinária e Zootecnia, Universidade de São Paulo (USP). Prof. Titular Aposentado do Departamento de Imunologia, ICB, USP.

Prof. Titular Visitante da Disciplina de Imunologia, Departamento de Microbiologia, Imunologia e Parasitologia, UNIFESP. Diretor Científico da Minipig, Pesquisa e Desenvolvimento Ltda. 
McClellan, 1965) $)^{3}$. Essas semelhanças são muito mais distantes quando a fisiologia do homem é comparada com aquela do cão, rato, camundongo e outras espécies utilizadas em experimentação. Em que pesem essas evidências, bem como aquelas que mostram a utilização de suínos de longa data na experimentação animal, o uso desta espécie como modelo experimental pela comunidade científica foi acanhado até os anos 70 .

A restrição do uso de suínos se prendeu à elevada capacidade de conversão alimentar com conseqüente ganho de peso desses animais. Nos dias atuais, onde o suíno foi transformado em verdadeira "fábrica" de conversão alimentar e conseqüente crescimento acelerado, após seis meses de vida pode atingir o peso de 150 quilos. Essas características fisiológicas da espécie, limitam o planejamento de experimentos em longo prazo. Por essa razão, suínos com essas características são utilizados em experimentos agudos ou de curta duração. Ainda, a utilização de animais muito jovens (leitões desmamados) induz a resultados não comparáveis com a fisiologia de humanos adultos.

\section{O miniporco}

As limitações descritas acima levaram cientistas a desenvolver colônias de suínos com dimensões compatíveis com a utilização dos mesmos em laboratório, facilitando assim o emprego destes animais como modelo de experimentação em diversas áreas de pesquisa médico-biológica. Esses animais foram denominados de miniporcos (minipigs) ou, dependendo das dimensões dos mesmos, suínos miniatura (miniature pigs).

O estudo programado para o desenvolvimento de uma população de suínos miniatura, iniciou-se no Instituto Homel da Universidade de Minnesota, em 1949 (England $e$ col. 1954) ${ }^{4}$. Resultados apresentados em
1965 e estudos nos 20 anos subseqüentes, substanciaram os objetivos de se produzir um animal de tamanho reduzido, anatômica e fisiologicamente normal. Publicações recentes indicam que a seleção genética para um tamanho reduzido continua em várias linhagens de suíno miniatura, incluindo o suíno Kangaroo Island do Sul da Austrália, o Minnesota, o Yucatan originário do sul do Mexico, o Goettingen produzido na Dinamarca e mais recentemente, a linhagem Sinclair (http://www.sinclairresearch.com/biotech.htm).

É importante salientar que o plano de desenvolvimento do minipig foi feito para produzir animais que, mesmo em idade adulta, sejam suficientemente pequenos para permitir sua utilização como animal de laboratório em pesquisas biomédicas. Esses animais não são portadores de nanismo.

Pode-se notar que o termo miniatura tem conotação de tamanho reduzido comparado com o suíno convencional. Os objetivos do desenvolvimento inicial determinaram o valor do suíno miniatura em duas categorias de tamanho, uma delas seria atingir um peso semelhante à média de peso do homem adulto $(70 \mathrm{a} 80 \mathrm{~kg})$ e outra de se conseguir um tamanho menor sem haver prejuízo dos aspectos fisiológicos e anatômicos normais na sua similaridade com o homem. Em 1981, a seleção genética para um reduzido tamanho, propiciou a obtenção de animais com 2 anos de idade com peso de 59,1 $\pm 5,2$ $\mathrm{kg}$ para o macho e $50,8 \pm 7,1 \mathrm{~kg}$ para fêmea. $\mathrm{Na}$ Universidade de Taiwan, a seleção intensiva produziu um animal com 12 meses com peso de 30,3 $\pm 2,6 \mathrm{~kg}$.

Em estudo realizado em rebanho-núcleo de suínos miniatura da Gottingen, Dinamarca (ellegaard@minipigs.dk), foram obtidos resultados que mostram as características biométricas desses animais em comparação com o suíno normal (Tabela 1).

TABELA 1 - Peso médio em kg, nas diferentes idades do suíno miniatura de Gottingen e do suíno normal.

\begin{tabular}{c|c|c}
\hline Idade em dias. & Suíno miniatura de Gottingen $(\mathbf{N}=\mathbf{2 0 4})$ & Suíno normal \\
\hline Nascimento & 0,542 & 1,40 \\
\hline 50 & 3,78 & 14,0 \\
\hline 70 & 8,60 & 30,0 \\
\hline 120 & 18,30 & 65,70 \\
\hline 165 & 25,10 & $\pm 100,00$ \\
\hline 214 & 33,30 & $\pm 120,00$ \\
\hline 260 & 41,20 & $\pm 150,00$ \\
\hline
\end{tabular}

A Tabela 2 mostra as características de outras linhagens estabelecidas de miniporcos e porcos miniatura. 
TABELA 2 - Características biométricas de algumas linhagens de miniporcos

(Fonte: http://www.criver.com/techdocs/swine.html).

\begin{tabular}{l|l|l}
\hline MINESOTA-Hormel & Tamanho da leitegada & 5,5 \\
& Peso ao nascer & $750-800 \mathrm{~g}$ \\
& Peso 140 dias & $18 \mathrm{~kg}$ \\
\hline PIMAN MOORE & Tamanho da leitegada & 6,0 \\
& Peso ao nascer & $670 \mathrm{~g}$ \\
& 2 a 3 anos de idade & $70 \mathrm{~kg}$ \\
\hline GOTTINGEN & Animal de 1 ano & $40 \mathrm{~kg}$ \\
\hline YUCATAN & Tamanho da leitegada & 6,12 \\
& Aos 6 meses & $20 \mathrm{~kg}$ \\
& Com 1 ano & $50 \mathrm{~kg}$ \\
& Com 2 anos & $83 \mathrm{~kg}$ (macho) \\
& & $70 \mathrm{~kg}$ (fêmea) \\
\hline
\end{tabular}

\section{O porco e o Miniporco nas diferentes áreas de pesquisa}

Segundo Bustard e McClellan (1965) o suíno, incluindo o miniporco, apresenta semelhanças com o homem no que diz respeito a aspectos ligados a odontologia, morfologia e fisiologia renal, acuidade visual, estrutura do olho, fisiologia e morfologia da pele, fisiologia e anatomia cardiovascular, fisiologia e anatomia digestiva e imunologia.

Tumbleson $(1986)^{5}$ complementa que o suíno é um modelo útil para estudos em pesquisas biomédicas, pois apresenta similaridade de estrutura e funções com o homem, incluindo tamanho, padrão de alimentação, fisiologia digestiva, hábitos dietéticos, estrutura e funções do rim, estrutura vascular do pulmão, distribuição da artéria coronária, propensão para a obesidade, taxa respiratória e comportamento social. Uma vez que o suíno é um onívoro, é considerado um modelo adaptável para avaliar as exposições crônicas e agudas ao álcool, cafeína, tabaco, aditivos alimentares e poluentes do ambiente. Esse animal tem sido usado com sucesso como modelo para avaliar alcoolismo, diabetes, absorção, nutrição parental total, transplante de órgãos, arteriosclerose, hipertensão, hemorragia, hipertensão, melanoma, gengivite, nefropatia obstrutiva e refluxo, osteocondrose e choque séptico.

\section{Trabalhos internacionais utilizando o miniporco}

A literatura referente ao uso do miniporco em pesquisa biomédica mostra a citação deste animal em mais de 5000 publicações nos últimos 30 anos. Como ilustra a Figura 1, a utilização desses animais como modelo experimental nas diferentes áreas da biomedicina teve início na década de 70 permanecendo em pequeno número até a década de 80 . Desse período em diante, o número de publicações/ano utilizando esses animais apresenta um crescimento contínuo.

Uma análise de regressão desses dados mostra que esse crescimento se faz segundo uma regressão linear, isto é, com tendência de aumento para os próximos anos.

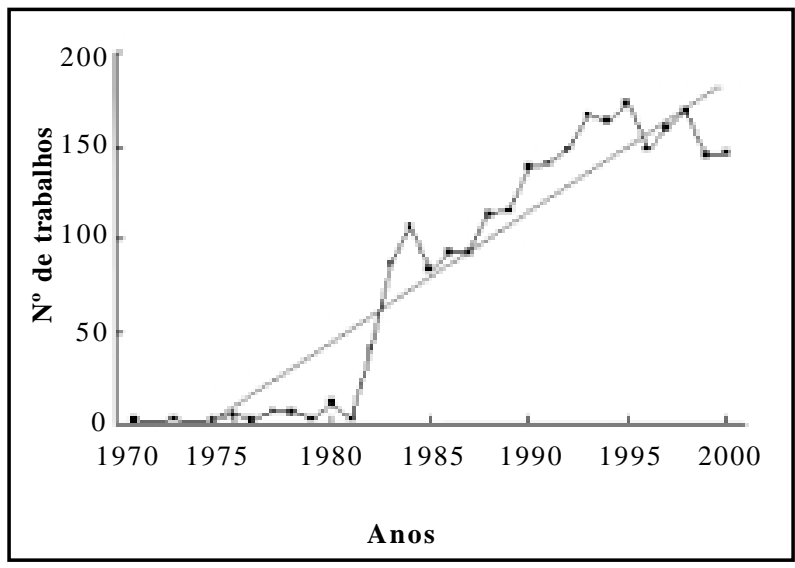

FIGURA 1 - Número de publicações que utilizam o minipig (PubMed).

\section{Utilização do miniporco no Brasil}

Em levantamento bibliográfico por nós realizado buscamos por "minipig AND Brazil". Como esperado, 
nenhuma publicação foi selecionada. Esse resultado se deve ao fato de que esse tipo de animal não é produzido em nosso país e ainda, que a importação dos mesmos demanda um investimento exorbitante. Para exemplificar, um miniporco com três meses de idade é vendido pela Gottingen (ellegaard@ minipigs.dk) por U\$ 800,00 sem a inclusão dos custos de transporte e importação.

\section{Do sonho à realidade}

Foi nos idos de 1964, quando aluno da Faculdade de Medicina Veterinária da USP e já engajado com a experimentação animal, que tivemos a oportunidade de ler artigo em revista (provavelemnte a revista Scientific American) que discorria sobre o desenvolvimento pelos americanos de uma colônia de suínos de pequeno porte destinados 'a experimentação. As justificativas para a seleção desses animais eram as mesmas acima expostas.

Tomados pelo entusiasmo procuramos pelos docentes ligados à suinocultura e seleção animal para que desenvolvessem projeto semelhante. Infelizmente, nosso entusiasmo não foi grande o suficiente para estimulá-los a embarcar no programa proposto.

Há sete anos, resolvemos retomar o projeto iniciando a seleção de suínos pelo tamanho com o intuito de estabelecer colônia de Minipigs a serem utilizados em experimentação por pesquisadores brasileiros.

Como resultado de dedicação e perseverança, está hoje constituída a empresa Minipig, Pesquisa e Desenvolvimento Ltda que conta com aproximadamente 100 animais com características de minipigs minipigs estes denominados Minipig brl. Esses animais são mantidos sob rigoroso esquema de higienização e alimentados com ração balanceada e adequada para as diferentes fases de crescimento. Todos os animais são sistematicamente vacinados contra Leptospirose, Erisipela Suína, Parvovirose, Rinite Atrófica e Pneumonia Enzoótica dos Suínos e, periodicamente, tratados com medicamentos para a prevenção de ecto e endoparasitos.

Atualmente a empresa inicia a seleção de linhagens homogêneas quanto ao peso corpóreo, teor de gordura, pelagem, cor e características de aprumo. Em que pese todas essas características não estarem ainda fixadas a principal delas, o peso corpóreo está controlado. Como mostra as Figuras 2 e 3, animais com 5 a 7 meses de idade (início da atividade sexual) pesam entre 10 a 15 quilos e animais com 12 meses de idade pesam entre 35 e 40 quilos permitindo assim que sejam utilizados em experimentos de longa duração.

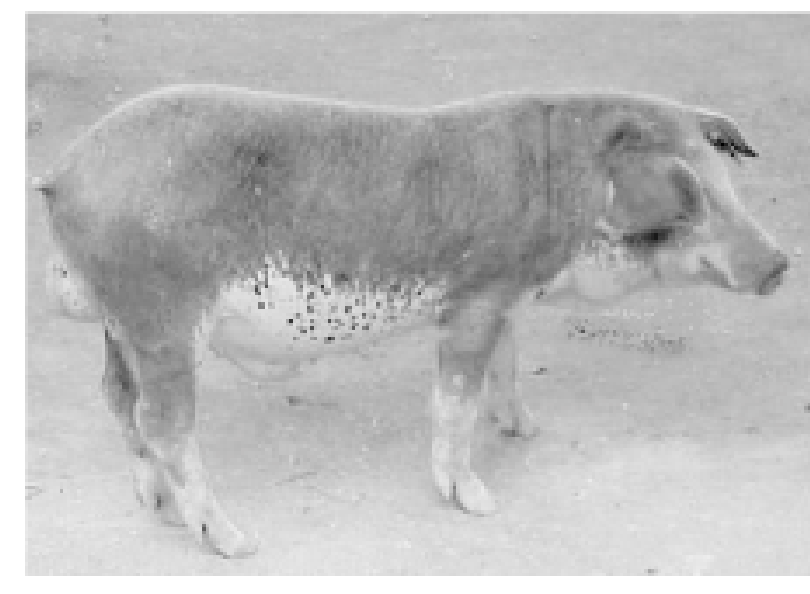

FIGURA 2 - Minipig $b r$, com 5 meses de idade, pesando 15 quilos.

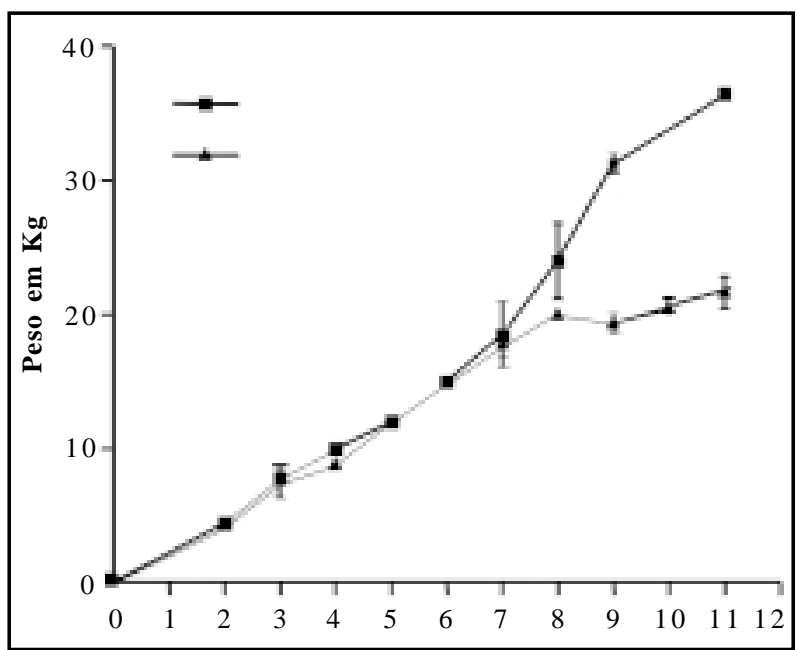

FIGURA 3 - Curva de ganho de peso de machos e fêmeas com no máximo 11 meses de idade.

Em pesquisa de opinião realizada pela Empresa foram entrevistados 18 pesquisadores que potencialmente poderiam vir a utilizar esses animais em seus projetos de pesquisa. Todos os entrevistados deram parecer favorável ao empreendimento com a ressalva, quase que unânime, sobre as possíveis dificuldades na manutenção e manuseio desses animais.

A fim de sanar tais dificuldades, a Empresa se associou com o Hospital de Equinos Salles Gomes, Foz e associados, localizada a 45 quilômetros de São Paulo, município de Jundiaí, que oferece o "homing" dos animais bem como toda a assistência veterinária altamente qualificada para a execução de experimentos da mais variada natureza nas áreas de anestesiologia, Raio$\mathrm{X}$, ultrasonografia e cirurgia geral. São acompanhados também projetos de longa duração tais como aterogê- 
nese, teratogênese e outros. Finalmente, a Minipig Pesquisa \& Desenvolvimento Ltda. tem condições de desenvolver ensaios toxicológicos para a avaliação de inocuidade e benefício de substâncias e produtos utilizados na industria.

\section{Perspectivas}

A importância da utilização de suínos na experimentação biomédica é claramente mostrada pelo número, qualidade e originalidade dos trabalhos realizados com esses animais em paises do hemisfério norte nos mais diferentes campos de experimentação. Com atraso de mais de 30 anos, esse modelo experimental certamente será utilizado por pesquisadores brasileiros colaborando assim para o maior desenvolvimento da ciência nacional.

\section{Referências}

1. Swindle MM. Basic surgical exercises using swine. Praeger Publishers; 1983.

2. Swindle MM, Moody DC, Phillips LD. Swine as models in biomedical research. 1ed. Ames, Iowa: Iowa State University Press; 1992.

3. Bustard LK, McClellan. Use of pigs in biomedical research. Nature 1965; 208:531-5.

4. England DC, Winters LM, Carpenter LE. The development of a breed of miniature swine: a preliminary report. Growth 1954;18:207.

5. Tumbleson ME. Swine in biomedical research. New York: Plenum Press; 1986.

Mariano M. Miniature swine (minipig) in biomedical experimental research: the Minipig brl. Acta Cir Bras [serial online] 2003 Sept-Oct;18(5). Available from URL: http://www.scielo.br/acb.

ABSTRACT - Different species of vertebrate and invertebrate animals have been used in experimental investigation. In this report a brief review on the use of swine in biomedical research and the similarities of the anatomy, physiology, biochemistry and immunology of this specie with those of man is presented. It is also reviewed the development in the 60th of the minipig and the achievements obtained with this animal model in distinct areas of experimental medicine. Finally, the basic characteristics of a colony of minipigs developed by the author are described. The conditions these animals are feed, housed, vaccinated and breed are described. They weight from 10-15 kilos when six months of age and from 30-37 kilos with 11 months of age. The possible contribution of this animal model for the development of experimental surgery and other areas of animal experimentation in this country is discussed.

KEY WORDS - Laboratory animals. Swine. Minipig.

Conflito de interesse: Divulgação Comercial Fonte de financiamento: Minipig, Pesquisa e Desenvolvimento Ltda

Correspondência:

Mario Mariano

Rua Leonardo Mota 66/83

05586-090 São Paulo - SP

Tels: (19)3252-3446 / 9138-0408

Fax: (19)3254-0377

mario.mariano@minipig.com.br www.minipig.com.br

Data do recebimento: 7/07/2003

Data da revisão: 21/07/2003

Data da aprovação: 4/08/2003 